\title{
Compression fatigue of a cellular Al alloy
}

\author{
Y. Sugimura ${ }^{\mathrm{a}, *}$, A. Rabiei ${ }^{\text {a }}$, A.G. Evans ${ }^{\mathrm{a}}$, A.M. Harte ${ }^{\mathrm{b}}$, N.A. Fleck ${ }^{\mathrm{b}}$ \\ ${ }^{a}$ Division of Engineering and Applied Sciences, Harvard University, 40 Oxford Street, Cambridge, MA 02138, USA \\ b Engineering Department, Cambridge University, Cambridge CB2 1PZ, UK
}

Received 15 July 1998; accepted 22 March 1999

\begin{abstract}
The cyclic compression of a cellular Al alloy has been evaluated. Plastic compression occurs beyond a critical number of cycles, $N_{\mathrm{T}}$. At $N<N_{\mathrm{T}}$ the cumulative strains are negligible and the material has cyclic stability. At $N>N_{\mathrm{T}}$, strain accumulates rapidly and preferentially within deformation bands, until the densification strain has been reached. The bands form preferentially from large cells in the ensemble. Such cells develop plastically buckled membranes which experience large strains upon further cycling, which lead to cracks. The cracks, once formed, result in rapid cyclic straining. This feature controls the fatigue life. C) 1999 Elsevier Science S.A. All rights reserved.
\end{abstract}

Keywords: Cellular Al alloy; Foam; Fatigue; Compression; Strain mapping

\section{Introduction}

Cellular Al alloys are ultralight compliant materials which, in compression, absorb considerable energy by plastic dissipation [1-8]. They are also resistant to chemical degradation, are flame retardant [9] and have high heat dissipation characteristics $[9,10]$. These properties suggest their implementation as durable strain isolation media. Their ability to perform in such applications is contingent upon longevity subject to cyclic compression. Accordingly, information about the fatigue $(S / N)$ response at strain/stress amplitudes below yield is essential. There are no such measurements in the open literature. This article addresses this deficiency by conducting cyclic compression tests, in conjunction with strain mapping, as well as by performing in-situ observations of cell deformations. These methods are used to identify the mechanisms and establish preliminary design criteria. A companion paper [11] provides further information. It examines the tension-tension as well as compression-compression cyclic properties of both open-cell and closed-cell aluminum alloy foams.

\footnotetext{
* Corresponding author. Tel.: + 1-617-496-3530; fax: + 1-617-4969771.

E-mail address: sugimura@esag.harvard.edu (Y. Sugimura)
}

This paper emphasises the behavior of one cellular $\mathrm{Al}$ alloy, designated ALPORAS [6]. This material has been selected because it is relatively homogeneous and has well-established monotonic mechanical properties [36]. Deformation bands begin forming at about $1 / 3$ to $1 / 2$ the plateau (peak) stress, designated $\sigma_{0}$ (Fig. 1) [5]. Some of these bands become inactive, others continue to compress, and additional bands form. The occurrence of plasticity at stresses above about $(1 / 2) \sigma_{0}$ suggests fatigue at peak stresses approaching this level. Cyclic tests conducted in this range comprise the primary measurement activity described in this article.

\section{Material and measurement methods}

The cellular aluminum alloy examined in this study is ALPORAS manufactured by Shinko Wire in Japan. The alloy consists of aluminum with $5 \mathrm{wt} . \%$ calcium and 3 wt.\% titanium from the manufacturing process. The average relative density, $\rho$, defined as the density of the foam divided by the density of the parent material, is 0.08 . The average cell wall thickness and the cell size are $85 \mu \mathrm{m}$ and $3 \mathrm{~mm}$, respectively. Detailed material characterization and mechanical properties of ALPORAS under static loading can be found in a previous study [4]. 


\subsection{Strategy}

Cyclic compression tests are performed, initially at a fixed $R$-ratio $(\mathrm{R}=|\sigma \min | /|\sigma \max |)$, while monitoring the development of cyclic deformation bands, as well as simultaneously following the deformation and fracture of cells located both within and outside the bands. Strain distributions are determined using a mapping procedure described elsewhere [5,15]. Cell deformation features are determined using two complementary methods:

1. Side surfaces suitable for optical imaging are prepared by electro-discharge machining (EDM). Oblique illumination is used to highlight the crosssection of the cell walls. Images are taken at several stages of fatigue, by periodically interrupting the test, and using identical imaging conditions each time. In some cases, scanning electron microscopy (SEM) is used to image the deformed cells.

2. Some specimens are sectioned by EDM along the loading axis after some fatigue has accumulated. A stamping procedure $[3,4]$ is used to provide vivid black and white contrast between the cell walls and the interior space. SEM images augment these findings.
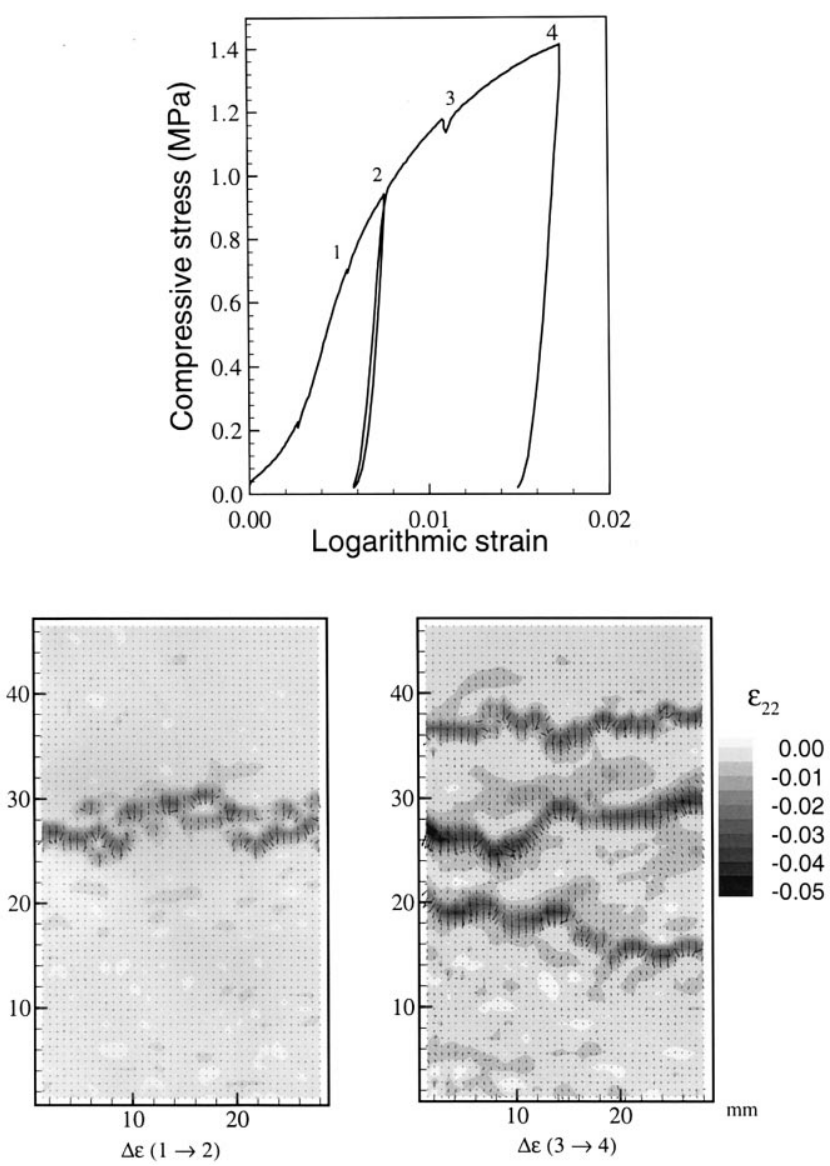

Fig. 1. Strain maps upon monotonic loading, indicating deformation bands [5].

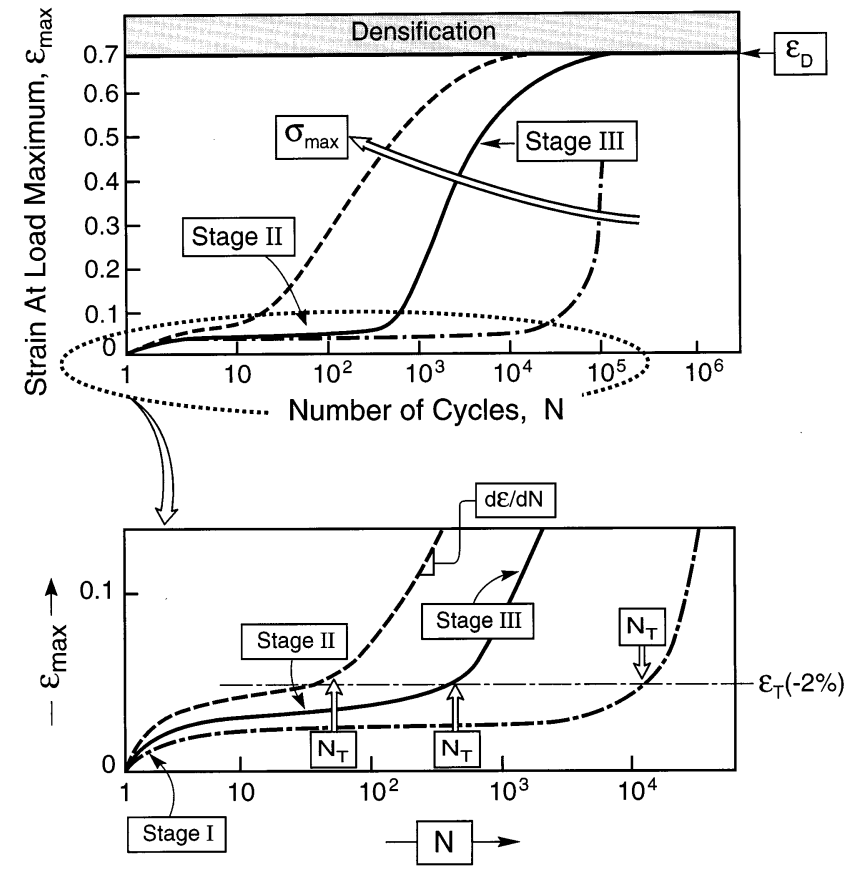

Fig. 2. A schematic summarizing the compressive fatigue behavior. Stage I involves small strains occurring in the first few decades. Stage II is a period of minimal strain accumulation, culminating at $N_{\mathrm{T}}$. Stage III is a domain of rapid strain accumulation until the densification strain is reached. The transition to stage III, at $N_{\mathrm{T}}$, occur at an essentially constant strain, $\varepsilon_{\mathrm{T}} \approx 2 \%$.

\subsection{Testing approach}

Compression specimens of the ALPORAS material have been prepared with dimensions $30 \times 30 \times 60 \mathrm{~mm}$ This size has previously been found large enough to avoid free-surface effects [3-5]. A compression alignment system has been used to apply axial loads [4]. Cyclic compressions are applied using a servohydraulic loading frame at $10 \mathrm{~Hz}$ with $R=0.1$. Strains are monitored using a displacement gauge attached to the central section with an adhesive and recorded digitally. Crosshead displacements are also recorded. The loaddisplacement information provides a permanent record that can be analyzed to determine cumulative strains, strain hysteresis and the elastic unloading modulus.

In some cases, the present results are compared with those from another batch of the material, reported elsewhere [11], which had somewhat different characteristics, related to superior material homogeneity.

\section{Deformation measurements}

Strain evolutions measured at constant load amplitude all have the features depicted on Figs. 2 and 3. These figures plot the strain measured at the stress maximum, $\varepsilon_{\max }$, as a function of the number of cycles, $N$, at several values of the maximum stress, $\sigma_{\max }$, for 
the fixed $R$-ratio and at the same frequency. The information is presented in schematic form in Fig. 2 in order to visualize the stages of deformation. The actual measurements are presented on Fig. 3. Following the inelastic strain in the first cycle, there is a small additional strain in the next decade, referred to as stage I. Thereafter, many cycles elapse with minimal further strain, designated the incubation period, or stage II. Then, at a critical number of cycles, $N_{\mathrm{T}}$, strain accumulates rapidly (stage III), until the material attains its densification strain, $\varepsilon_{\mathrm{D}}$ (Figs. 2 and 3 ).

The onset of stage III, at $N_{\mathrm{T}}^{*}$, is strongly affected by $\sigma \max$ (Fig. 3). ${ }^{1}$ The consequent $S / N$ plots (Fig. 4a) reveal a substantial difference in fatigue between this material and another batch [11]. However, upon normalizing by $\sigma_{0}$, the results for both batches superpose (Fig. 4b).

Two results obtained at $\sigma_{\max }=0.85 \mathrm{MPa}$ (Fig. 3) reveal a decade difference in $N_{\mathrm{T}}$. This difference reflects the stochastic nature of the deformation of these materials, also apparent from the monotonic measurements [5].

During stage III (the strain accumulation phase) there are often two or more abrupt strain jumps, illustrated by arrows on Fig. 5. The strains associated with each jump are of order $5 \%$. These features are more vividly illustrated by replotting the measurements as the increase in strain-at-maximum-stress, per cycle, $\mathrm{d} \varepsilon / \mathrm{d} N$, as a function of $N$. Such results for one test at $\sigma_{\max }=0.85 \mathrm{MPa}$ (Fig. 6) reveal four key characteristics.

1. In stage I, the strain per cycle decreases systematically from $\sim 10^{-3}$ to $\sim 10^{-7}$.

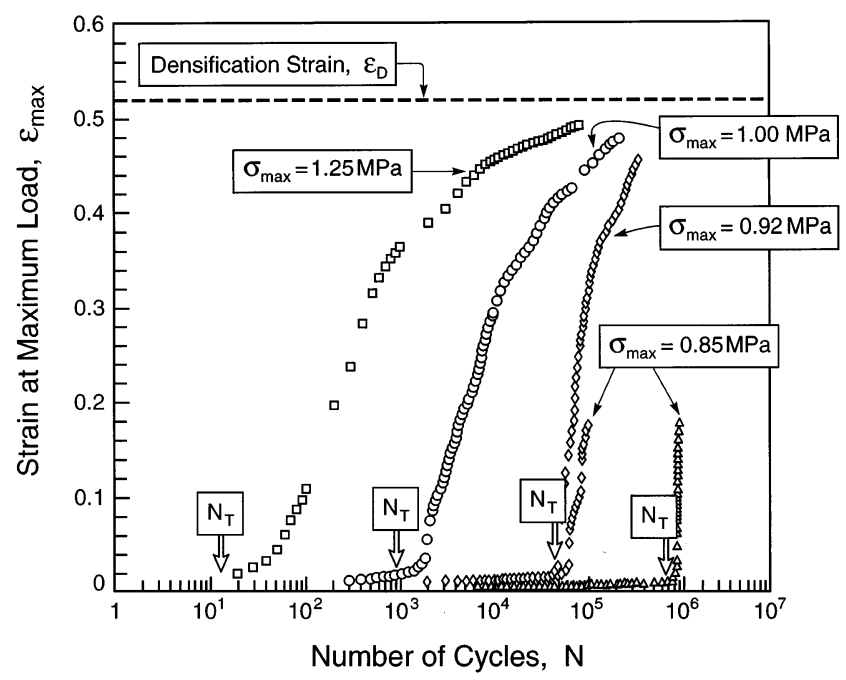

Fig. 3. The strain, $\varepsilon_{\max }$, at the load maximum, plotted as a function of the number of fatigue cycles at several different stress maxima, $\sigma_{\max }$, with $R=0.1$ at a frequency of $10 \mathrm{~Hz}$. Logarithmic cycles are used to show the full range of measurements, with $N_{\mathrm{T}}$ indicated.

\footnotetext{
${ }^{1}$ The transition is so abrupt that any strain level between 2 and $10 \%$ gives essentially the same $N_{\mathrm{T}}$.
}

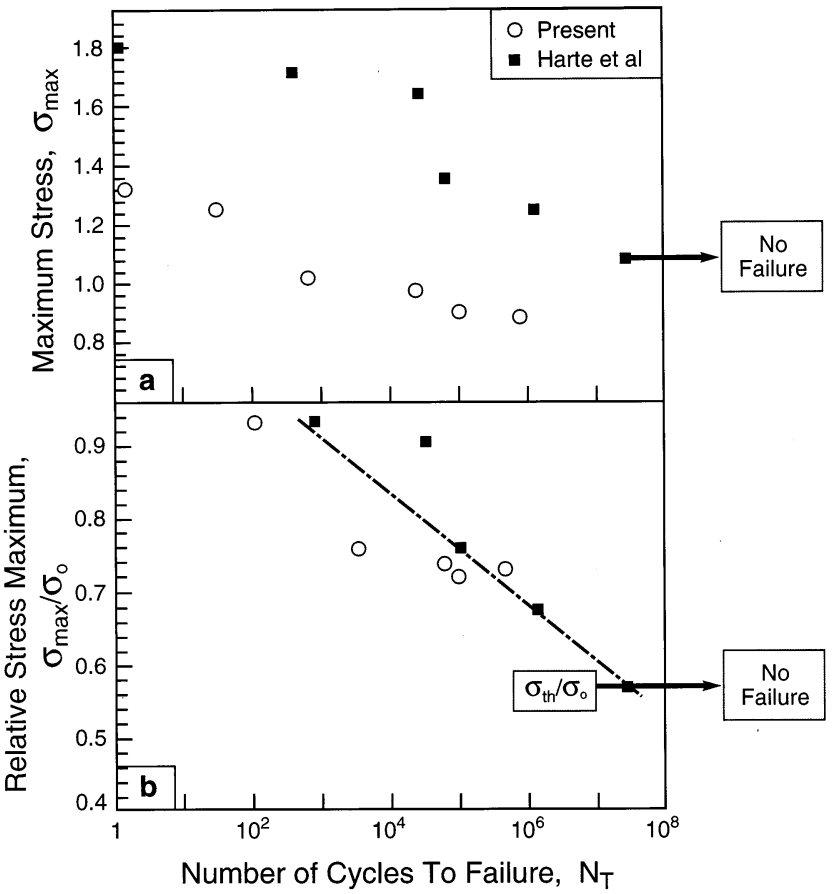

Fig. 4. Compression $S / N$ curves expressed as the effect of stress maximum, $\sigma_{\max }$, on the number of cycles to the stage II/III transition, $N_{\mathrm{T}}$, regarded as the fatigue life. (a) Based on the absolute stress, $\sigma_{\max }$. (b) Corresponding plots using the relative stress maximum, $\sigma_{\max } / \sigma_{0}$.

2. In stage II, $\mathrm{d} \varepsilon / \mathrm{d} N$ remains constant at an imperceptibly small level, of order $10^{-7}$.

3. The onset of stage III, at $N_{\mathrm{T}}$, coincides with a large jump in $\mathrm{d} \varepsilon / \mathrm{d} N$ to about $10^{-4}$.

4. Within stage III, strain accumulates at $\mathrm{d} \varepsilon / \mathrm{d} N$ between $10^{-5}$ and $10^{-6}$. But, there are further strain jumps similar to that at $N_{\mathrm{T}}$. More uniform strain rates have been found in the less heterogeneous material [11].

By replotting $\mathrm{d} \varepsilon / \mathrm{d} N$ against strain, $\varepsilon_{\max }$ (Fig. 7), it becomes apparent that the first strain jump occurs at essentially the same strain level for all stress maxima.

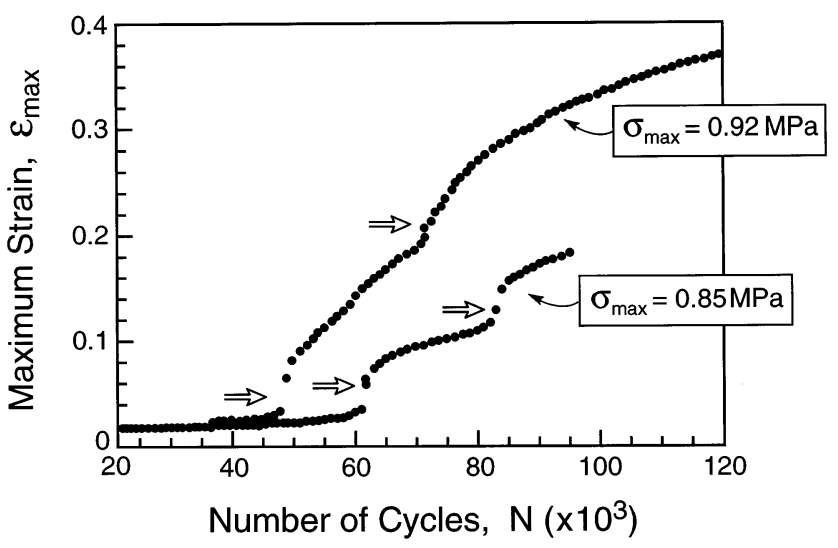

Fig. 5. The strain in stage III at two load maxima highlighting the strain jumps (horizontal arrows). 


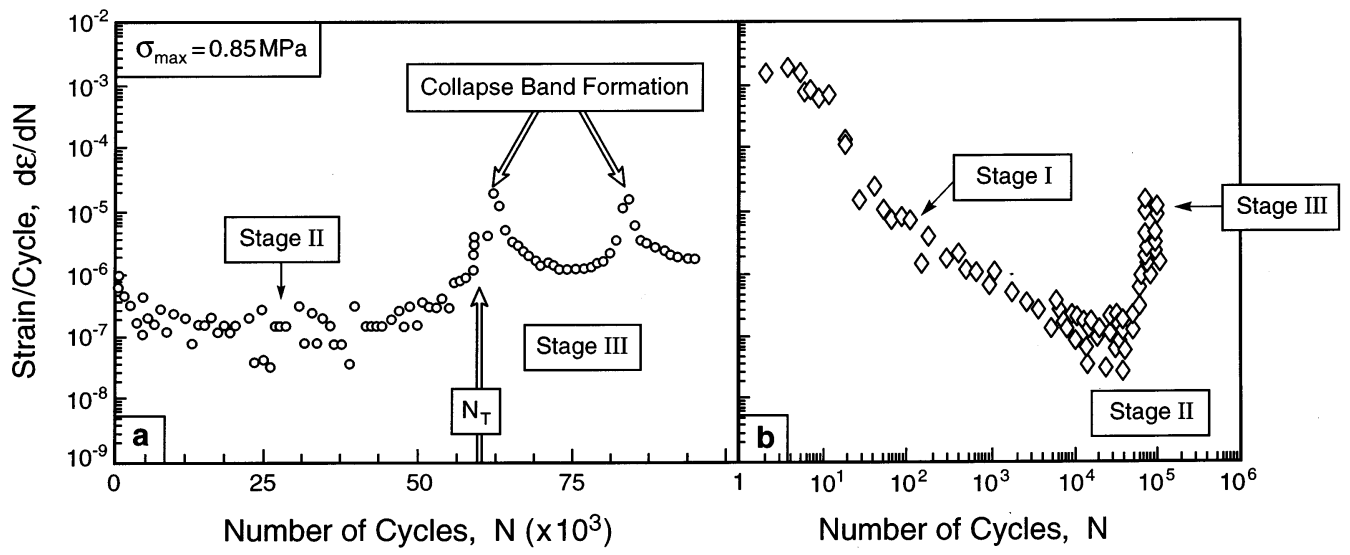

Fig. 6. A replot of the data for one of the specimens tested at $\sigma_{\max }=0.85 \mathrm{MPa}$, using the strain-at-maximum stress, per cycle, d $\varepsilon / \mathrm{d} N$, as the ordinate. (a) Linear plot that emphasises stages II and III highlighting the uniformity of strain-rates in stage II, as well as the strain jumps in stage III, at $N_{\mathrm{T}}$ and beyond. (b) Logarithmic plot that emphasises the decaying strain-rate in stage I.

The first occurs at a strain of $\sim 5 \%$ and the second in the range $12-20 \%$. In all cases, there is a minimum in $\mathrm{d} \varepsilon / \mathrm{d} N$ at a strain of $2 \%$. This latter finding is the basis for ascribing the onset of stage III to a fixed strain, designated $\varepsilon_{\mathrm{T}}$ on Fig. 2. It will be shown below that each jump commences with the formation of a cyclic deformation band.

When $N>N_{\mathrm{T}}$, the hysteresis increases as cycling proceeds and the unloading modulus decreases.

\section{Strain fields}

Strain mapping indicates that deformation bands form in the first cycle (Fig. 8), consistent with findings upon monotonic loading [5]. Upon unloading from the stress maximum, further plastic deformation occurs (Fig. 8), indicative of reverse yielding. The unloading deformations are on planes that, mostly, disconnect from those responsible for the deformations found at the stress maximum. Further study is needed to quantify and understand this behavior.

Important insight has been gained by monitoring the evolution of the strain distribution at peak stress, as a function of the number of stage II cycles (at $N \geq N_{\mathrm{T}}$ ). The results for one such test with $\sigma_{\max }=0.92 \mathrm{MPa}$ are presented on Fig. 9. The greatest accumulation of normal strain, $\varepsilon_{y y}$, occurs at three sites, denoted A, B and $\mathrm{C}$ in the figure, having dimensions of order the cell size. Moreover, appreciable strains occur on planes intersecting these locations, oriented about normal to the loading axis. These are the planes that exhibit abrupt strain jumps in stage III, at $N \geq N_{\mathrm{T}}$. The band of high strain that forms around the site designated A on Fig. 9 was present after the first cycle. It began as a small cell-sized domain with excess strain. Upon continued cycling, this domain expanded into a band while the strain level increased. Conversely, the site of the second band, denoted $\mathrm{C}$, had no evident strain excess after the first cycle. It emerged later and became prominent after $10^{4}$ cycles. It intensified upon further cycling.

\section{Cell morphologies}

Observations made using optical and scanning electron microscopies are presented at increasing levels of detail, subject to the finding from the strain mapping that deformation bands indicate from discrete, cellsized sites ( $\mathrm{A}$ and $\mathrm{C}$ in Fig. 9) and then experience

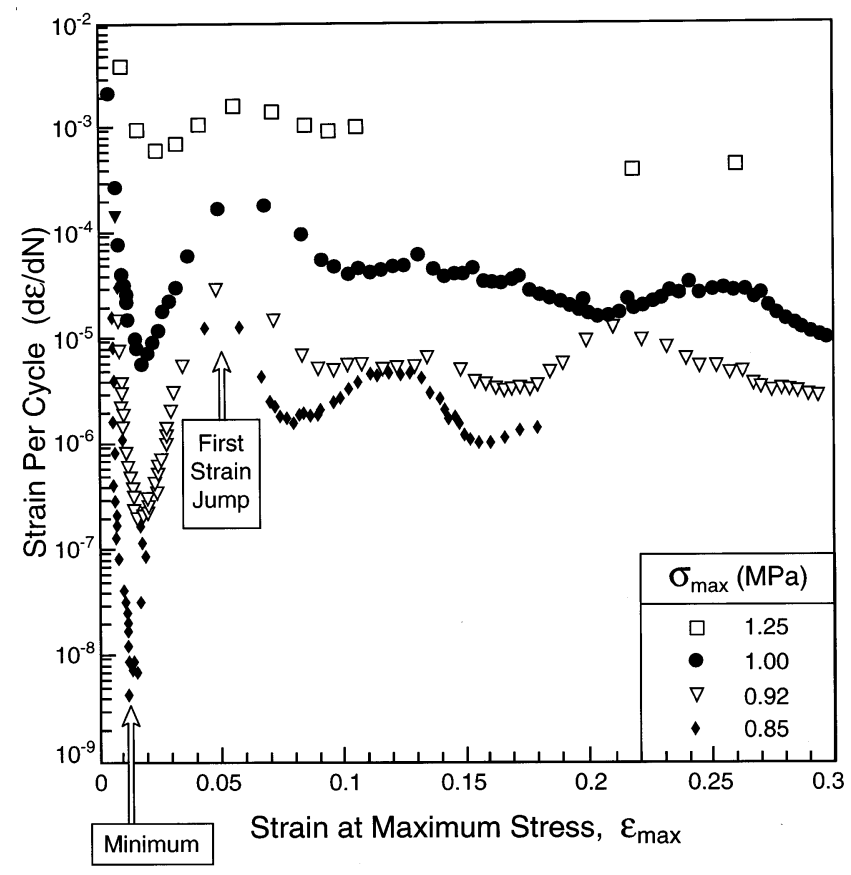

Fig. 7. The data from Fig. 6 replotted as $\mathrm{d} \varepsilon / \mathrm{d} N$ against cumulative strain, $\varepsilon_{\max }$. In all cases, there is a minimum at $2 \%$ strain and moreover, all strain jumps occur at strains of $\sim 5 \%$ (first) and $12-20 \%$ (second). 

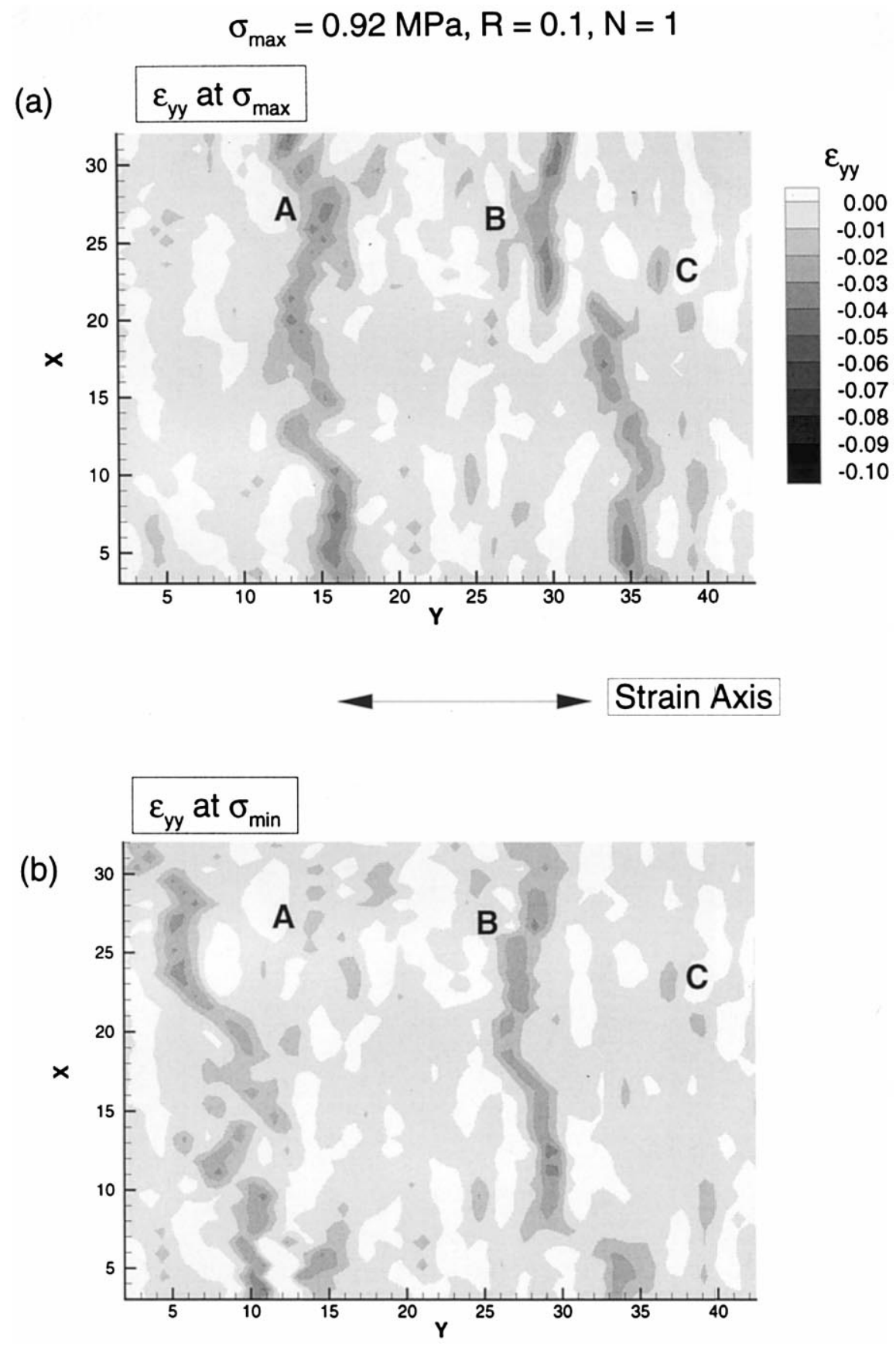

Fig. 8. Strain maps obtained during the first cycle: (a) at the stress maximum; and (b) at the stress minimum. The stress level in this test was 0.92 MPa. The strains $\varepsilon_{y y}$ are along the $y$-axis, coincident with the loading.

densification. For preliminary characterization purposes, one of the specimens tested into stage III $(18 \%$ strain) was cross-sectioned through the center (Fig. 10). Two distinct deformation bands $\left(\mathrm{AA}^{\prime}\right.$ and $\left.\mathrm{BB}^{\prime}\right)$ are evident from the disruption of the cellular morphology. Outside these bands, there are no obvious changes.

Direct before-and-after optical images of side surfaces provide additional information. By examining the bands on orthogonal side faces, the normals to the bands were found to exhibit a range of orientations $\beta$ relative to the loading axis, $30^{\circ} \geq \beta \geq 0^{\circ}$. The bands often intersect, suggesting that the first band may be responsible for subsequent bands.

The morphological changes occurring within the bands are illustrated by means of sequential images of the same surface taken at different stages in the fatigue process (Fig. 11). Images are shown in the unloaded state after strains of zero (the reference state), $2 \%$ (the culmination of stage II), $8 \%$ (after the first strain jump into stage III) and 15\% (after the second strain jump within stage III). 

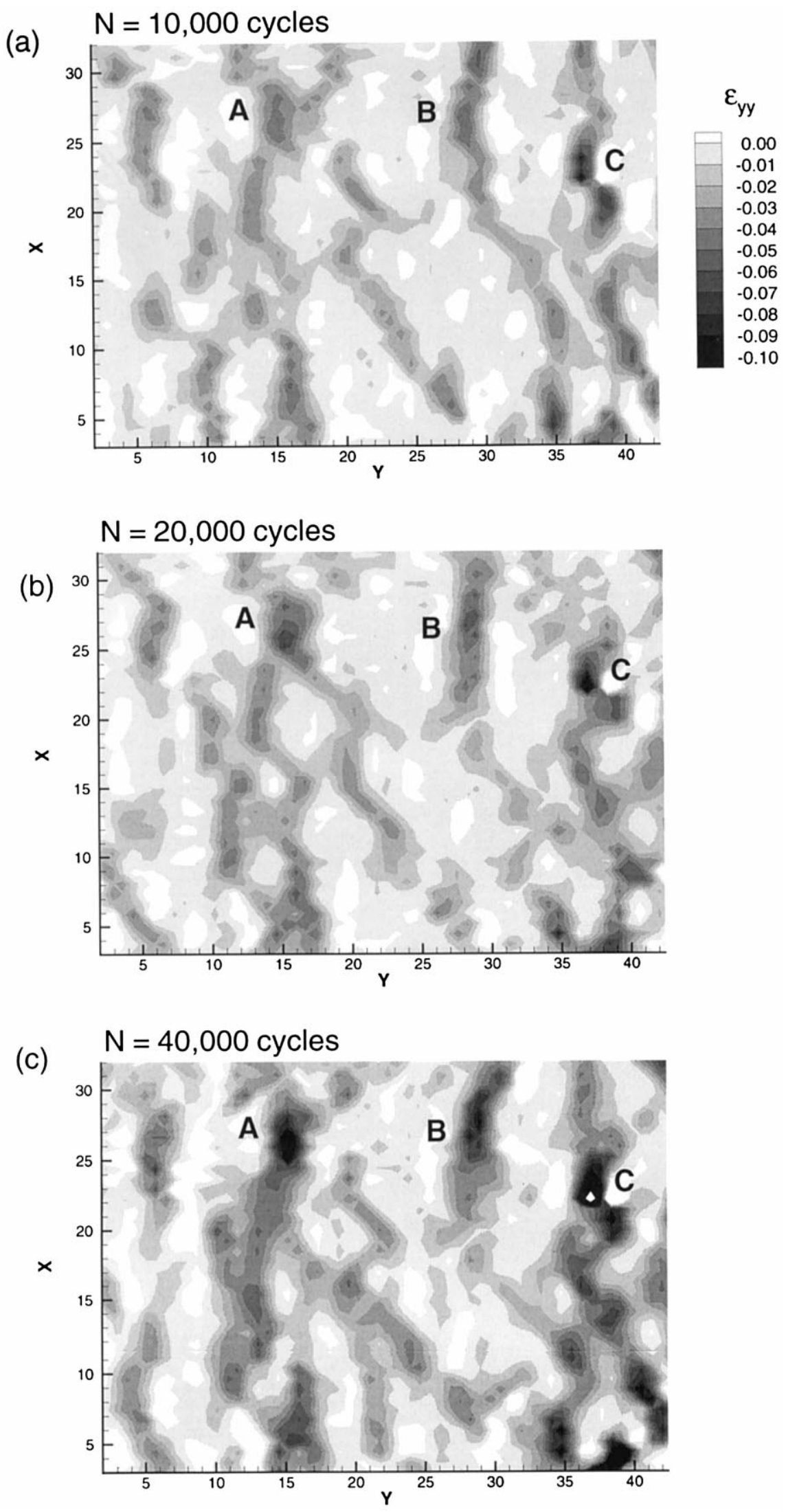

Fig. 9. Strain maps at the stress maximum $\left(\sigma_{\max }=0.92 \mathrm{MPa}\right)$ at various stages during stage II: (a) 10000 cycles; (b) 20000 cycles (c) 40000 cycles. The latter is just before the abrupt stress jump into stage III. The three sites of maximum strain accumulation are identified as A, B and C. 
At $2 \%$ strain (Fig. 11c) one of the circled membranes next to the largest cell (identified as cell A) has buckled plastically. After the first strain jump (Fig. 11d) a single collapse band becomes evident, indicated by the arrows. It occurs at precisely the location of the large cell (A) containing the buckled membrane (Fig. 11c). By comparing the length of this large cell (along the $y$-axis), both before (Fig. 11b) and after (Fig. 11d) band formation, the displacement associated with the band may be determined. It is $\sim 3 \mathrm{~mm}$. This displacement is equivalent to the average size of the cells located along the band trajectory. Accordingly, most cells fully densify when the band forms, subject to displacements normal to its plane. At this strain, there is retrospective evidence of local plastic buckling of another circled membrane at a second large cell, identified as cell B (Fig. 11d). This membrane is also identified in Fig. 11c. Note that, in the as-manufactured state (Fig. 11b), this membrane contains a single wiggle (apparent from the bright contrast).

After $15 \%$ strain, following the second strain jump (Fig. 11e), a second band is evident, identified with arrows. Moreover, it occurs around cell $\mathrm{B}$ at the buckled membrane identified on Fig. 11c and d. The associated displacement is again $\sim 3 \mathrm{~mm}$, normal to the plane of the band.

Accordingly, both bands originate at large cells. Such cells experience plastic buckling in one constituent membrane within stage I or II. Subsequently, when each band forms, it collapses completely, with accompanying displacements about equal to the typical cell size along its prospective plane. These findings are consistent with those ascertained from strain mapping (Fig. 9).

A

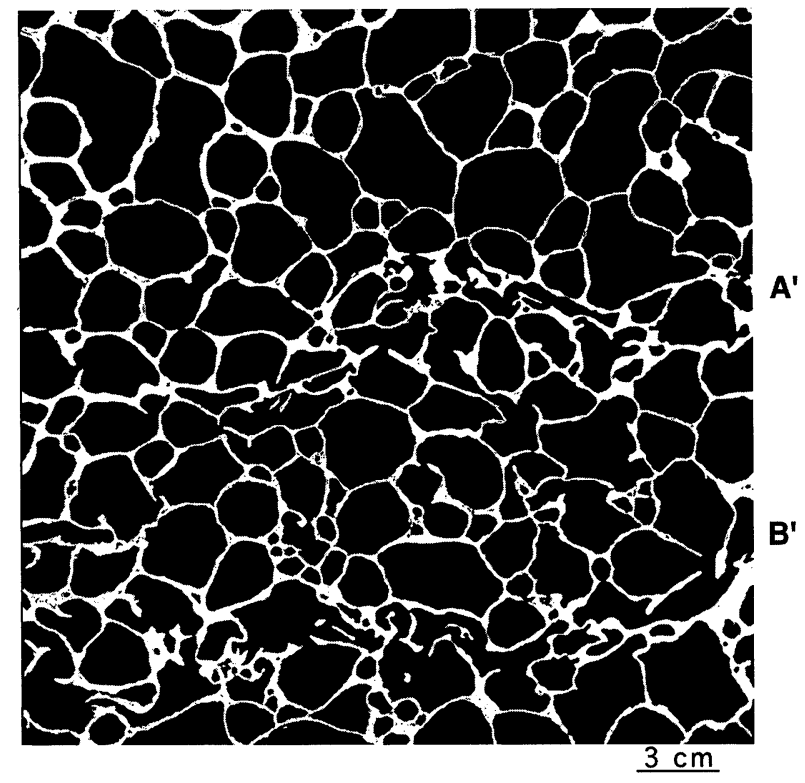

Fig. 10. Optical, stamped image on an axial section after an applied strain of $18 \%$ (after formation of the second collapse band). The two bands are located between $\mathrm{AA}^{\prime}$ and $\mathrm{BB}^{\prime}$.
Corresponding observations made on the more homogeneous material [11] suggest that a single band forms at $N_{\mathrm{T}}$ which thereafter, broadens to encompass the gauge section.

\section{Cell damage}

Determination of the softening mechanisms that lead to collapse requires images at yet higher resolution. These images are obtained using both optical and scanning electron microscopies.

The optical images taken after the second band has formed (Fig. 12a), reveal that the cells within the bands are fully buckled. Moreover, many of the cells in the region between the bands have become substantially distorted. There is evidence of plastically buckled and bent membranes in various stages of displacement. A membrane crack is also apparent in at least one of the cells (Fig. 12b).

The cracks are more completely characterized by SEM imaging (Fig. 13). These images reveal that, within the collapse band, the membranes are not only distorted, but contain a high density of ruptures, with appreciable opening displacements. They are similar in appearance to cell wall cracks found both upon tensile testing of this material and upon monotonic compression [4]. Some cracks originate at small holes that pre-exist in the cell walls.

\section{Mechanisms}

The fatigue response in this material is unusual by virtue of the long incubation stage [14]. For implementation purposes, the mechanisms that dictate the number of cycles at the stage II/III transition, $N_{\mathrm{T}}$, are most important. The challenge is that $N_{\mathrm{T}}$ coincides with the incidence of the first fully developed band.

Gradual changes occurring in stage II (Fig. 9) comprise the softening mechanism. Since cracks are evident within the bands (Fig. 13), it is surmized that these are the source of the softening mechanism. That is, cracks grow in some cell membranes during stage II and, when large enough, initiate plastic collapse of the cells within the band, by reducing the membrane contribution to the plastic resistance. A proposed mechanism is depicted on Fig. 14.

Fatigue is introduced by initial plastic buckling of one or more membranes. In some case, this happens in the first cycle, with an apparent preference for the largest cells. ${ }^{2}$ Some further straining occurs in the next few

\footnotetext{
${ }^{2}$ This differs from monotonic yielding which occurs preferentially of cells with high ellipticity [5].
} 

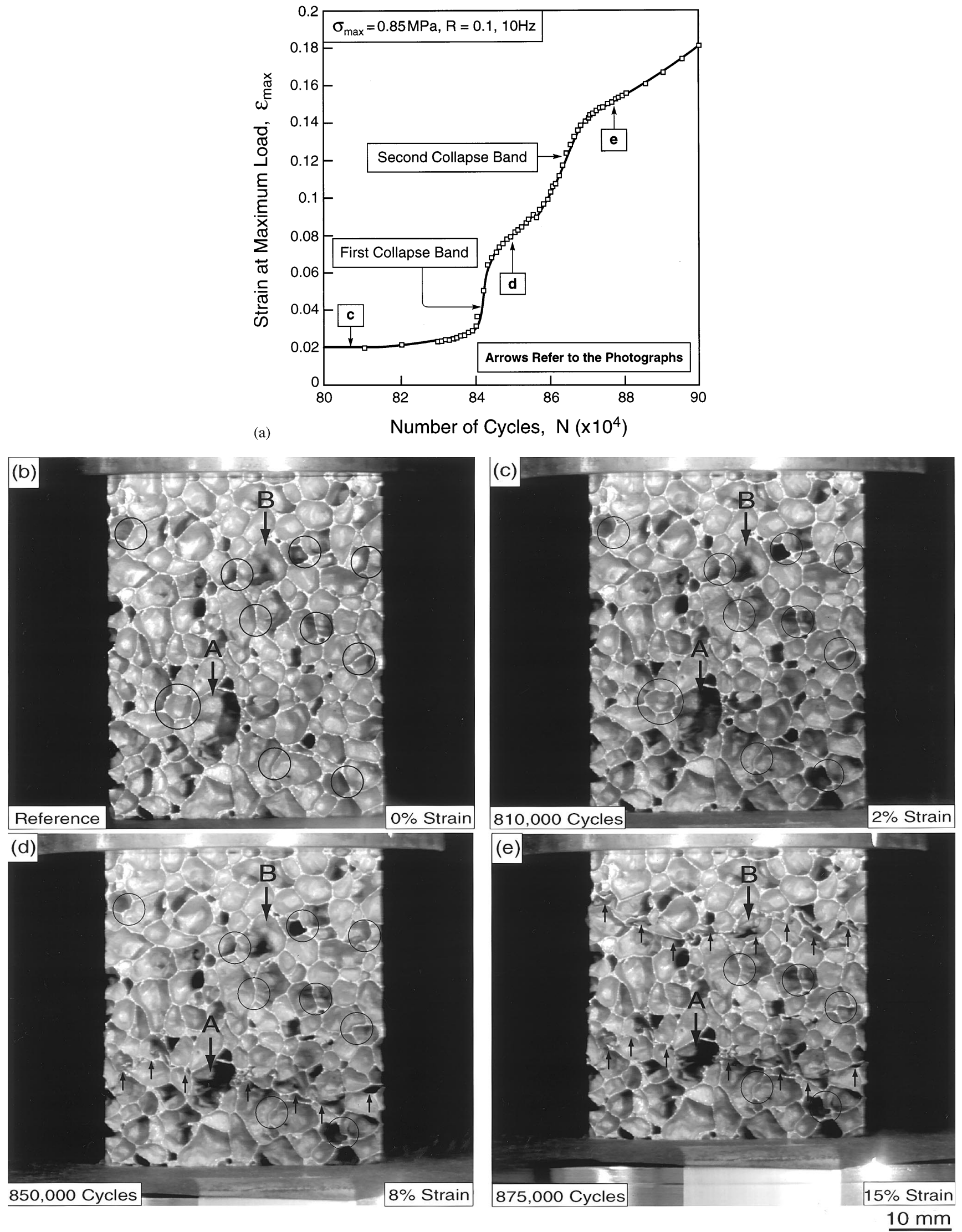

Fig. 11. Sequential optical images of the cyclic deformations at: $\Delta \sigma / \sigma_{0} \approx 0.65$ : (a) the strain curve indicating the number of cycles at which three of the images were taken. (b) Image before testing, (c) within the incubation stage, $2 \%$ strain, (d) after the first strain jump; $8 \%$ strain, (e) after the second strain jump, $15 \%$ strain. The circles identify the membranes that buckle between (b) and (c). The arrows highlight the collapse bands. 


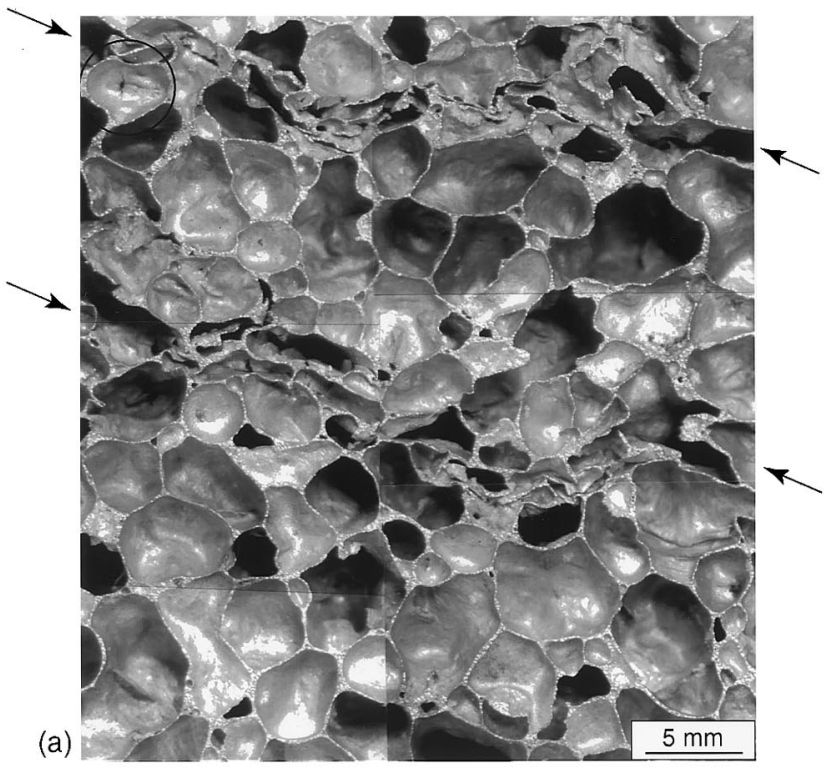

(b)

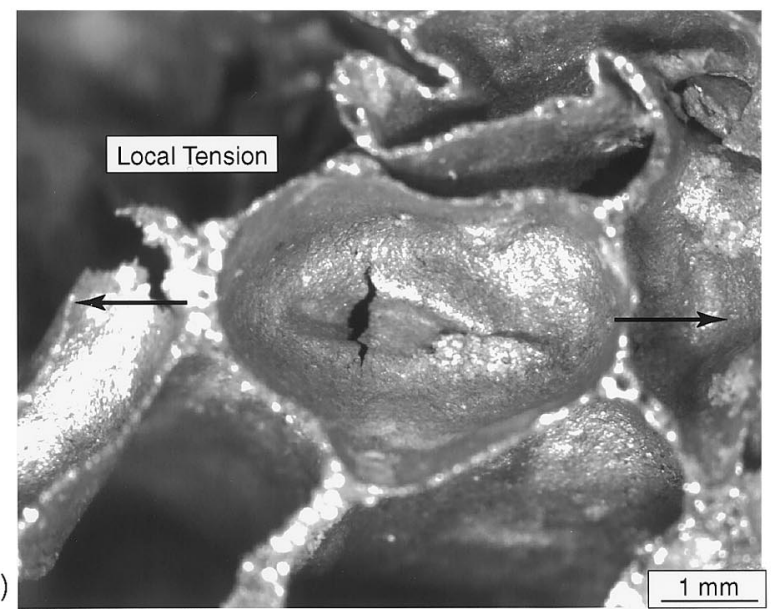

Fig. 12. (a) An optical image of two cyclic deformation bands indicating the nature of the cell deformations within the bands. (b) A membrane crack formed near one of the deformation bands.

decades (stage I) but quickly saturates, indicative of shakedown. This happens because the deformations are localized and elastically constrained.

The phenomena occurring around the plastically buckled membranes are considered to be the source of band formation and fatigue failure. That is, cyclic strains that occur in the regions around the buckled membranes have exaggerated amplitudes that lead to crack formation. These strain amplitudes are manifest in hysteresis loops. Once such crack form, the associated stress redistribution results in unstable collapse within the contiguous cells. Such a mechanism would be characterized by a Coffin-Manson law [11-14] operating locally in the highly strained membranes.

Yet, the effect of stress amplitude on $N_{\mathrm{T}}$ (Fig. 4) is much greater than that associated with the conventional Coffin-Manson law, which has a logarithmic slope of $\sim 1 / 2$. It is surmized that the local strain amplitudes around the buckled membranes are strongly affected by the stress maximum, through the magnitude of the plastic buckling introduced in the first cycle, with the following consequences. There is a threshold $\sigma_{\text {th }}$ which occurs at stresses below that needed to cause plastic buckling in the first cycle. The present results (Fig. 4) suggest that: $\sigma_{\text {th }} / \sigma_{0} \approx 0.65$. Between $\sigma_{\text {th }}$ and $\sigma_{0}$, there is a rapid change in the severity of buckling as $\sigma_{\max }$ increases. This causes the cyclic strain amplitude to increase appreciably, resulting in a fatigue life strongly dependent on $\sigma_{\max }$. The details have yet to be established.

When normalized by the monotonic plateau stress, $\sigma_{0}$, the $S / N$ curves (Fig. 4) are unified. The basic mechanism leading to formation of the first cyclic deformation band thus appears to be insensitive to material heterogeneity. However, the subsequent behavior, $N>N_{\mathrm{T}}$, is variable. Materials with lower $\sigma_{0}$ are more stochastic in nature.

\section{Conclusion}

Cellular Al alloys have a relatively well defined fatigue life in cyclic compression. It is associated with an abrupt increase in strain after $N_{\mathrm{T}}$ cycles. This event is governed by a single cyclic deformation band. This band completely densifies with an associated displacement of $\sim 3 \mathrm{~mm}$, equal to the cell size. Further cycling either causes additional bands to form or induces band spreading until full densification occurs.

The bands that arise at $N \geq N_{\mathrm{T}}$ originate from plastically buckled membranes that form much earlier in the fatigue process: in some cases in the first cycle. Moreover, these buckled membranes appear to arise preferentially at the largest cells in the medium. Accordingly, the fatigue life may be connected to the presence and distribution of large cells. The exaggerated cyclic strains that occur in those membranes encompassing the buckled morphology are subject to gradual crack formation. Once formed, the cracks cause sufficient softening that the associated deformation band collapses and densifies, resulting in the strain jump at $N_{\mathrm{T}}$. The details of crack evolution have yet to be worked out. A model of this process would be needed to establish a fatigue lifing capability for these materials. 

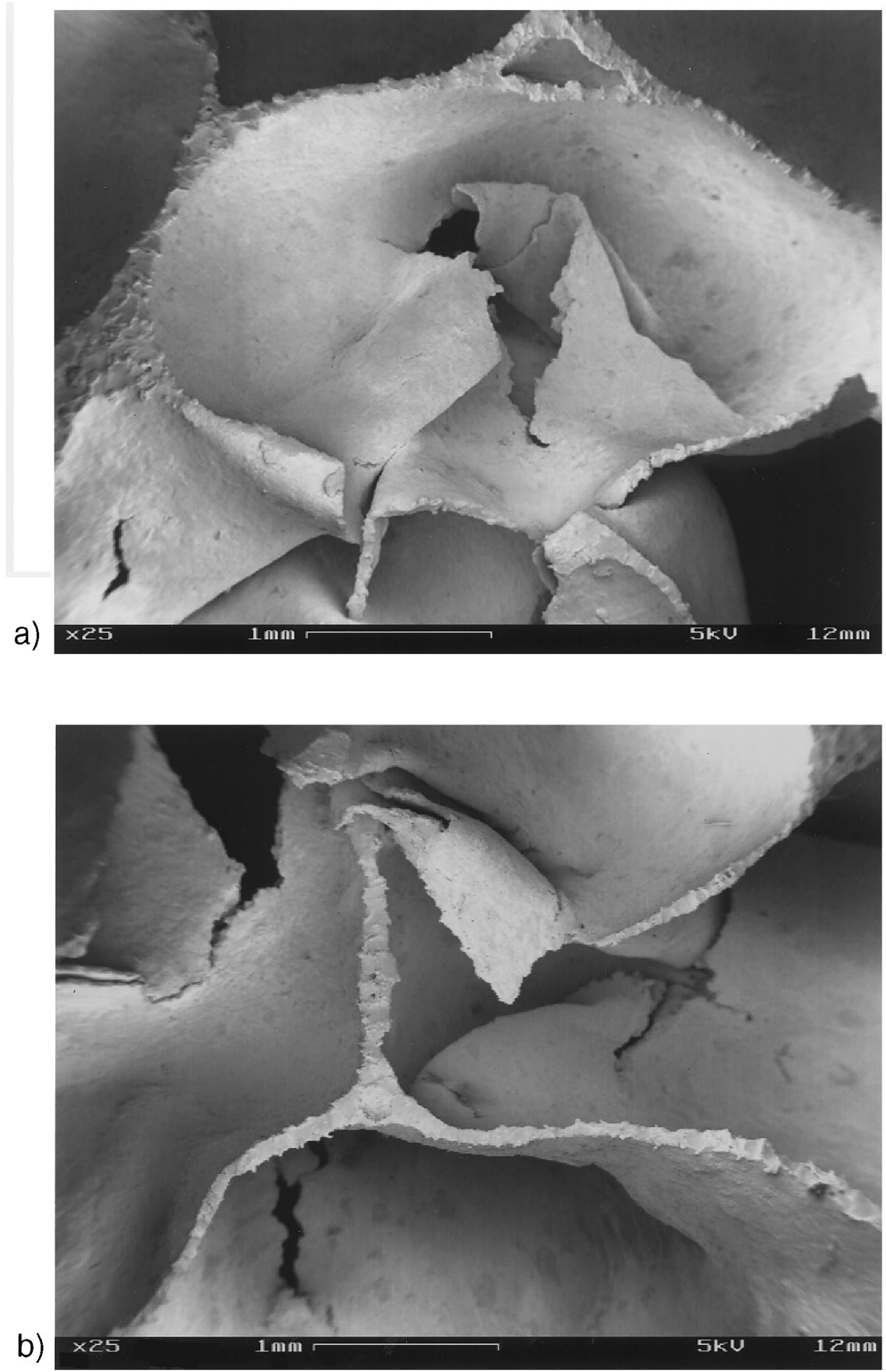

Fig. 13. Scanning electron microscope images of cells after $18 \%$ strain accumulation in stage III. Cells within the deformation bands reveal plastic buckling of some membranes, as well as numerous cracks. 


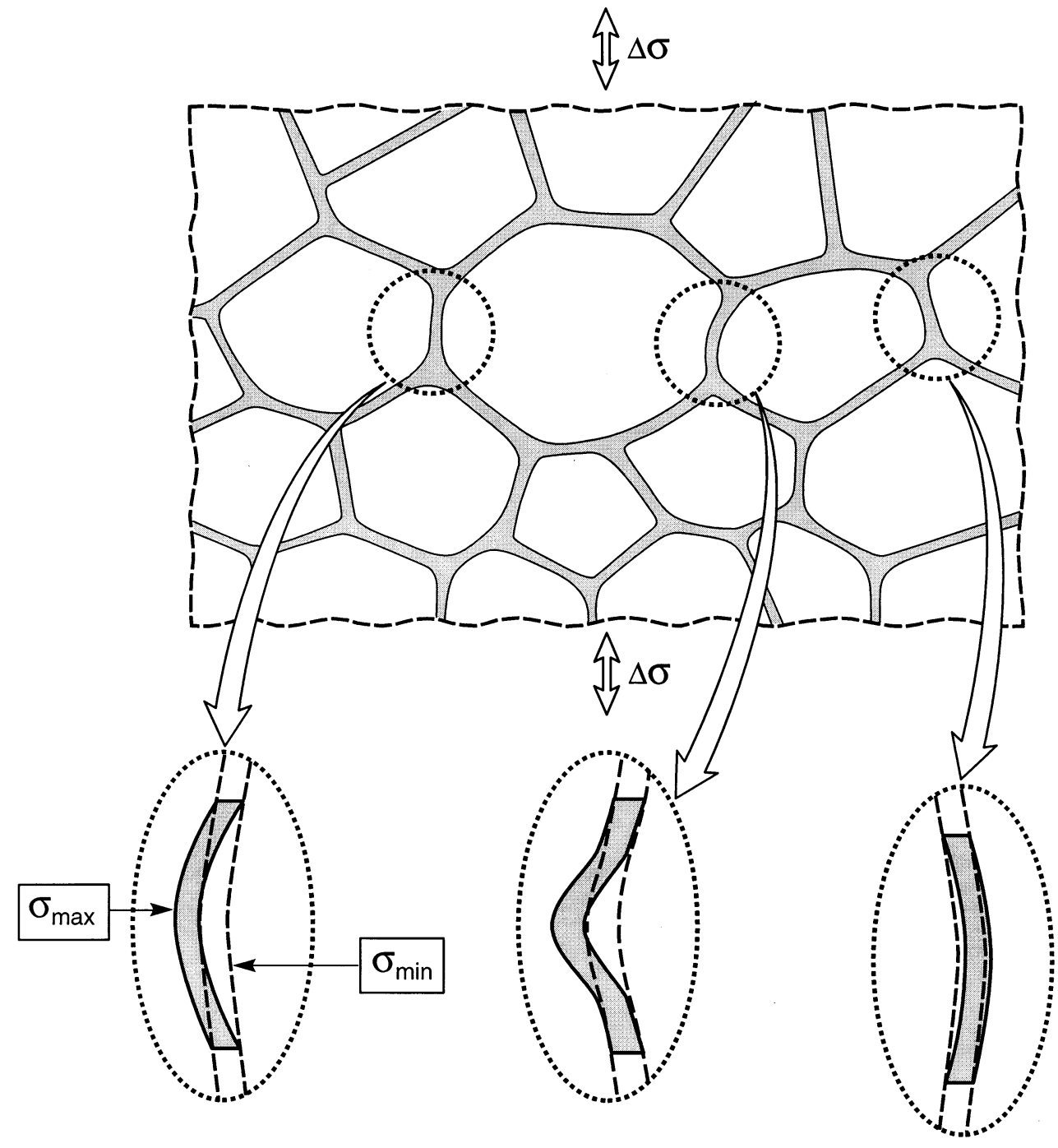

Fig. 14. A schematic of the proposed fatigue mechanism operating at large cells in the ensemble.

\section{References}

[1] L.J. Gibson, M.F. Ashby, Cellular Solids, 2nd edition, Cambridge University Press, Cambridge, UK, 1997.

[2] A.G. Evans, J.W. Hutchinson, M.F. Ashby, Progress in Materials Science 43 (1998) 171.

[3] A.E. Simone, L.J. Gibson, Acta Mater. 46 (1998) 2139.

[4] Y. Sugimura, J. Meyer, M.Y. He, H. Bart-Smith, J.L. Grenestedt, A.G. Evans, Acta Mater. 45 (1997) 5345.

[5] H. Bart-Smith, A.F. Bastawros, D.R. Mumm, A.G. Evans, D.J. Sypeck, H.N.G. Wadley, Acta Mater. 46 (1998) 3583.

[6] S.Akiyama, K. Imagawa, A. Kitahara, S. Nagata, K. Morimoto, T. Nishikawa, M. Itoh, Foamed Metal and Method for Producing Same, US Patent No. 4,712,277 (1987).
[7] P.H. Thornton, C.L. Magee, Met. Trans. 6A (1975) 1801.

[8] J. Beals, M. Thompson, J. Mater. Sci. 32 (1997) 3595.

[9] T.J. Lu, H.A. Stone, M.F. Ashby, Acta Mater. 46 (1998) 3619.

[10] A. Bastawros, H.A. Stone, A.G. Evans, J. Heat Transfer, submitted for publication.

[11] A.M. Harte, N.A. Fleck, M.F. Ashby, Acta Mater., in press.

[12] L.F. Coffin, Trans. ASME 76 (1954) 931.

[13] S.S. Manson, National Advisory Commission on Aeronatucs: Report 1170, Lewis Flight Propulsion Laboratory, Cleveland, $\mathrm{OH}, 1954$.

[14] S. Suresh, Fatigue of Materials, Cambridge University Press, Cambridge, 1991.

[15] A.F. Bastawros, H. Bart-Smith, A.G. Evans, JMPS, in press. 